See discussions, stats, and author profiles for this publication at: https://www.researchgate.net/publication/339324790

\title{
Modified Local Ternary Pattern Based Face Recognition using SVM
}

Research · July 2019

CITATIONS

0

3 authors, including:

Raja K B

University Visvesvaraya College of Engineering

188 PUBLICATIONS 1,341 CITATIONS

SEE PROFILE

Some of the authors of this publication are also working on these related projects:

Biometrics View project

Biometrics View project
READS

245 


\title{
Modified Local Ternary Pattern Based Face Recognition using SVM
}

\author{
Pattarakamon Rangsee ${ }^{1}$, K B Raja ${ }^{2}$ and Venugopal K R ${ }^{3}$ \\ ${ }^{1,2}$ Dept. of Electronics and Communication Engineering, University Visvesvaraya College of Engineering, \\ Bangalore University, Bangalore, India \\ ${ }^{3}$ Vice-Chancellor, Bangalore University, Bangalore, India \\ pattarakamon@gmail.comªnd Raja_kb@yahoo.com²
}

\begin{abstract}
Face recognition (FR) has drawn considerable interest and attention in the area of pattern recognition. FR is still a challenging task in real time applications even though they are a number of face recognition algorithms which are available and work in various constrained environment. The paper proposes a FR algorithm using Modified Local Ternary Pattern (MLTP) with multi class Support Vector Machine (SVM) classifier. The MLTP features of the face images are classified by an Error-Correcting Output Code (ECOC) multiclass model with SVM. The proposed method is tested on six standard face databases. The experimental results have been demonstrated that the performance of MLTP with SVM can achieve higher recognition accuracy compared to the conventional methods.
\end{abstract}

Keywords- Local Binary Pattern (LBP), Local Ternary Pattern (LTP), Support Vector Machine (SVM), Face Recognition

\section{INTRODUCTION}

With the rapid development of security technologies, biometric recognition systems are widely used in numerous application systems due to its high recognition accuracy and, since they are very convenient for users and tough to steal. Biometrics is the science of recognizing individuals which can be divided into two groups based on the characteristics like behavioral and physiological. The behavioral characteristics are related to walking style, way of speaking, keyboard typing, handwriting etc. The physiological characteristics are related to the shape and appearance of the body like face, fingerprint, palm print, iris, DNA etc. The fingerprint recognition is the traditional method of biometric which is commonly used in various applications. Another method which is based on iris tissues is iris recognition, the most secure system but very expensive. In order to overcome the drawbacks of fingerprint and iris recognitions, face recognition is used because of universality, reliability, and user friendly nature.

Faces are the most commonly used biometric for identification and authentication to recognize the authorized persons. The Human Computer Interaction (HCI) based face recognition is either feature based or image based approach. In recent years, there are many papers on feature extraction based on face recognition for both software developments and applications as discussed in the literatures [1]- [5].

Many feature extraction techniques for face recognition have shown higher recognition accuracy by using different machine learning algorithms like SVM, decision tree, random forest, etc. It shown that SVM classifier has significantly improved the performance of the recognition rate compared to state-of-the-art methods.

This paper presents a unique face recognition system with Modified Local Ternary Pattern (MLTP) using SVM classifier. The proposed algorithm uses Modified Local Ternary Pattern for feature extraction. The resultant final feature vector of training dataset and test dataset are compared using an ECOC multiclass model with SVM classifier. The predicted output is tested with the trained feature and test feature, resulting in high accuracy.

The organization of the paper is as follows. The literature survey for feature extraction and different classification techniques are described in section II. In section III, background such as various face databases, LBP, LTP and SVM are explained in detail. The proposed model is discussed in section IV. The performance analysis of the proposed algorithm is applied and compared with existing algorithms are tabulated in section V. Finally, the conclusions are discussed in section VI.

\section{RELATED WORK}

While this section is intended to give state-of-the-art review of facial feature extraction methods, this work concentrates on local feature based methods and classification methods that are considered in machine learning as a support vector machine. A large portion of this section's content is hence mainly focused on the above discussed approach.

Naoufel Werghi et al., [1] have presented a popular method using LBP which is applied on a mesh for $3 \mathrm{D}-\mathrm{FR}$. To extract the feature vectors, firstly computation of LBP descriptors is done using both shape as well as with photometric information of the face mesh surface. Then, grids are constructed to set the regions on face images. The histogram is applied on each region and concatenated to obtain structured encoding for both partial and whole face image. Jiwen $\mathrm{Lu}$ et al., [2] have proposed a Simultaneous Local Binary Feature Learning and Encoding (SLBFLE) method. Further, they have proposed a Coupled-SLBFLE (C-SLBFLE) for heterogeneous face matching, which is performed by using collective structured and latent feature learning to minimize the heterogeneous gap among the face images of dissimilar modalities. Byungyong Ryu et. al., [3] have proposed a face descriptor for facial expression recognition, a Local Directional Ternary Pattern (LDTP). This method uses encoded information features which are used to obtain the guiding information and ternary pattern to proceed with higher

978-1-5386-7516-8/18/\$31.00 C2018 IEEE 
edge responses in the boundaries of the facial features and by eliminating the unused from regions in the totaled directional patterns. Yao $\mathrm{Wu}$ and Weigen Qiu [4] have proposed enhanced Local Ternary Pattern (LTP) integrated with Stacked Auto-Encoder (SAE). The enhanced LTP extracts the features of the face and SAE is used for classification. The experimental results on $\mathrm{CK}+$ database have shown that the enchanced LTP data which is integrated with SAE method significantly increases the facial expression recognition rate. Swati Manhotra and Reecha Sharma [5] have proposed a novel method of facial feature extraction with illumination normalization. This method is used as a gradient based illumination normalization technique in the pre-processing stage, and then LBP and LTP are used which are efficient methods for insensitive to monotonic grayscale variations in the image as a feature extraction. Highly correlated facial image features were extracted while removing the irrelevant features by applying LBP to monotonic grayscale variations and LTP to make more noise resistant. The facial feature extraction process is performed on two popular face databases, Extended YALE B and AR database. The feature vectors obtained from LBP and LTP are highly discriminative and are useful for further recognition tasks. Hamdi Dibeklioglu and Theo Gevers [6] have proposed spontaneous approximation of the test images from the face images in videos. Deep learning method is used for regional face dynamic per frame and are encoded to fisher vector per region to define the video. Then the obtained regional fisher vector is concatenated and thus classified using linear SVM. The experimental results show high accuracy for the proposed work. Anima Majumder et. al., [7] have presented deep network outline for FR system. The method consists of four geomatics feature extraction, regional LBP feature extraction, combination of both LBP and geomatics feature extracted by auto encoders and using both SVM and Kohonen Self Organizing Map (SOM)-based classifier for classification. The performance of the proposed method using fused feature extraction and SOM based classifier were compared using SVM classifier. Santosh Tirunagari et.al., [8] have proposed classification pipeline which consist of Dynamic Mode Decomposition (DMD), LBP and SVM with histogram kernel. The distinct property of DMD is that, it can denote the temporary data of whole video as a single image with same dimensions as the image contains in that video. The method is tested on publicly available database and demonstrates the efficiency and effectiveness of the model. Shilpa Sharma and Kumud Sachdeva [9] have proposed a Principle Component Analysis (PCA) system by using SVM and Speed Up Robust Feature (SURF) for face recognition. The method is invariant to posture, brightening and maturing in particular PCA then they used SVM to classify the recognition capacity. The matching score of the source images are coordinated with the database. The results show higher accuracy and yields in better mean square error.

\section{BACKGROUND}

In the previous section, different feature extraction and classification techniques were discussed. One of the popular feature extraction technique is texture descriptor which represents texture in numerical form by giving importance to unique properties of texture. Local Binary Patterns (LBP) is a basis gray scale invariant texture descriptor which gives high performance by encoding the neighborhood into binary patterns. In this section, details of different face database, the detailed understanding of LBP and its extension and LTP are provided in sections $\mathrm{A}, \mathrm{B}$ and $\mathrm{C}$, respectively. In section $\mathrm{D}$, the powerful classification technique, Support Vector Machine (SVM) is discussed in brief.

\section{A. Databases}

To evaluate the performance of the face recognition, various standard face database are used to check for the efficiency and reliability of the proposed algorithm. A good database should capture image of each person under variant conditions with many facial expressions and different poses. In this paper, the standard face databases such as AT\&T (formerly ORL) database, YALE, Indian Females, Indian Males, JAFFE and NIR are considered to estimate the accuracy and compute the performance of the proposed model.

1) The ORL Database of Faces [10]: One of the most important publicly standard face database known as AT\&T ("The ORL Database of Faces") contains a set of face images that consists of total 400 images from 10 distinct images of each of 40 different persons. For few people, the images are captured under different sessions of diverse illumination, with different facial details such as wearing glasses or not and various facial expressions such as opening of eyes, closing of eyes, etc. Each face image in the database has a size 112 X92 pixels.

2) The YALE Database of Faces [11]: The Yale database has totally 165 grayscale images with .gif format (originally) from 11 distinct images of each of 15 different persons. The face images of each person are captured with different facial expression with occlusions, and are also taken under different illuminations and face angles. Each face image in the database has a size $243 \mathrm{X} 320$ pixels.

3) The Indian Female Database of Faces [12]: The Indian Female face database has totally 242 images from approximately 12 distinct images of each of 20 different persons. Each image of one person has various facial orientations and expressions with smile, laughter, sad, disgust and neutral on the white background. Each face image in the database has a size 480 X640 pixels.

4) The Indian Male Database of Faces [13]: The Indian Male face database has totally 220 images from approximately eleven distinct images of each of 20 different persons. Each image of one person has various facial orientations and expression same as the Indian Female face database on the white background. Each face image in the database has a size $480 \times 640$ pixels.

5) The JAFFE Database of Faces [13]: The Japanese Female Facial Expressions (JAFFE) has totally 213 grayscale images with TIFF format from 10 different persons. Each image of one person is based on 7 different facial expressions which includes unhappiness, shock, happiness, fear, anger,

\section{- IEEE}


repulsion and neutral movements. Each face image in the database has a size $256 \times 256$ pixels.

6) The NIR Database of Faces [14]: The Near Infrared (NIR) face database has totally 1800 images from fifteen distinct images of each of 120 different persons. In each image, the person has variations in expression, pose and scale. And the images of some persons are taken in different times. Each face image in the database has a size 576X768 pixels.

\section{B. Local Binary Pattern (LBP):}

It is one of the most popular features in face recognition literature. In basic LBP, the whole image is divided into sub blocks of size $3 \times 3$ pixel value and LBP is applied to each sub block to extract the LBP feature. In each sub block, the center pixel value $\left(x_{c}, y_{c}\right)$ is considered as a threshold to compare with its eight neighbor pixel values. The $\operatorname{LBP}_{\mathrm{P}, \mathrm{R}}$ operator can be expressed as in Eq. (1) and (2)

$$
\operatorname{LBP}_{P, R}\left(x_{c}, y_{c}\right)=\sum_{n=0}^{P-1} S\left(f_{n}-f_{c}\right) 2^{P}
$$

where $f_{n}=$ neighbor pixel intensity value.

$f_{c}=$ central pixel's intensity value $\left(x_{c}, y_{c}\right)$.

$P=$ the number of neighboring pixel values of the center pixel.

$R=$ the radius of circle around the center pixel value to corresponding neighbors.

$$
S\left(f_{n}-f_{c}\right)= \begin{cases}0, & f_{n}-f_{c}<0 \\ 1, & f_{n}-f_{c} \geq 0\end{cases}
$$

If the pixel value of the neighbor pixel is greater than or equal to the center pixel, one (' 1 ') is assigned to that pixel but zero (' 0 ') is assigned when pixel value of the neighboring pixel is less than the center. Then the LBP code is generated for the center pixel by concatenating eight zeros or ones to a binary code, and then the binary code is converted into decimal number. This decimal number represents the LBP number for each sub block $3 \mathrm{X} 3$ as show in Fig. 1.

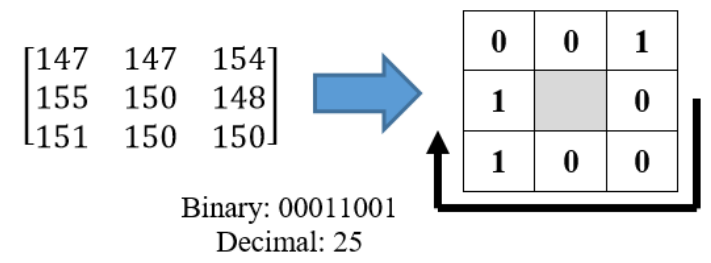

Fig. 1. The LBP Operator

In additional, the increasing size of neighborhood is called extension LBP (ELBP). The radius $R$, increases from the center pixel. The number of neighbors are equal to $8,24,48$ $\ldots$ and $R$ is equal to $1,2,3 \ldots$, respectively.

\section{Local Ternary Pattern (LTP):}

Local Ternary Pattern is applied for pattern recognition for feature extraction. It is an extension of LBP as it is sensitive to noise. For LBP, if the central pixel has changed then the codes for neighborhood in an image may also change, and is observed especially for smooth regions. LTP is used to solve the drawback of LBP by improving the threshold function around zero to assess the difference in the local grayscale. LTP is an extended version of basic LBP with three-value codes. In LTP, the whole image is divided into sub blocks of size $3 \times 3$ pixel value and LTP is applied to each sub block to extract the LTP feature. In each sub block, $\mathrm{f}_{\mathrm{c}}$ is the center pixel value $\left(x_{c}, y_{c}\right)$ and is considered as a threshold to compare with eight neighbor pixel values $f_{n}$ and $I$ is a user-defined threshold. The $L T P_{P, R}$ operator can be expressed as in Eq. (3) and (4)

$$
\operatorname{LTP}_{P, R}\left(x_{c}, y_{c}\right)=\sum_{n=0}^{P-1} S\left(f_{n}-f_{c}\right) 2^{P}
$$

where $f_{n}=$ neighbor pixel intensity value.

$f_{c}=$ central pixel's intensity value $\left(\mathrm{x}_{\mathrm{c}}, \mathrm{y}_{\mathrm{c}}\right)$

$P=$ the number of neighboring pixel values of the center pixel.

$R=$ the radius of circle around the center pixel value to corresponding neighbors.

$$
S\left(f_{n}-f_{c}\right)=\left\{\begin{aligned}
-1, & f_{n}<\left|f_{c}-I\right| \\
0, & \left|f_{c}-I\right|<f_{n}<\left|f_{c}+I\right| \\
1, & f_{n}>\left|f_{c}+I\right|
\end{aligned}\right.
$$

If the pixel value of the neighbor pixel is greater than the center pixel plus threshold $(\mathrm{fc}+\mathrm{I})$, then one is assigned to that pixel. If the pixel value of the neighbor pixel is equal to the threshold length, then zero is assigned to that pixel but minus one is assigned when pixel value of the neighbor pixel is less than the center pixel minus threshold (fc-I).

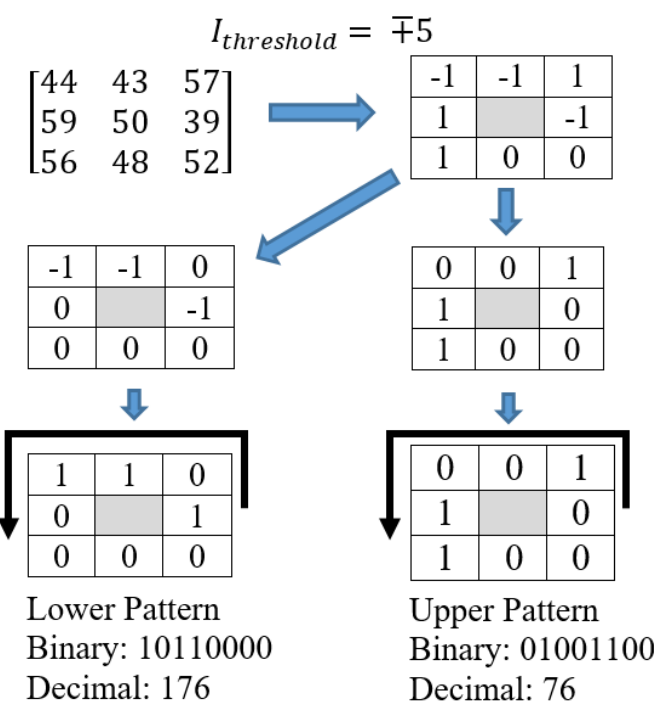

Fig. 2. The LTP Operator 
The 3 X3 matrix with values of zero's, minus one's and one's are generated based on Eq. (4). The $3 \times 3$ matrix is converted into two $3 \mathrm{X} 3$ binary matrices viz., lower pattern matrix by assigning minus one's by one's and other values by zero's and upper pattern matrix retain one's by assigning zero's to other values are shown in Fig. 2.

\section{Support Vector Machine (SVM)}

It is the most popular and widely used machine learning algorithm for classifier. The classifier separates the hyperplane used in the classification of face images for successful face recognition. SVM is a supervised learning method, given learning data, the algorithm outputs the ideal hyperplane which sets the novel examples. The algorithm is essentially designed for binary classification of the face images. The two dimensional hyperplane is a line which is divided into two classes where the information can be on any of the side. SVM can also be used for multi-class classification in many of the wider applications like pattern recognition with different models. The data is split into training and test dataset. The training dataset is set for higher dimensional space resulting in separates classes for more than one object. If the hyperplane is set back to the original dimensional space, then it results in nonlinear classifier. Therefore, to obtain ideal hyperplane, the SVM should achieves a better training algorithm to reduce the error function.

\section{Proposed Face ReCognition Model.}

In this section, a face recognition model based on Modified Local Ternary Pattern (MLTP) for feature extraction and SVM as a classifier is proposed. The structure of the proposed model is as shown in Fig. 3.

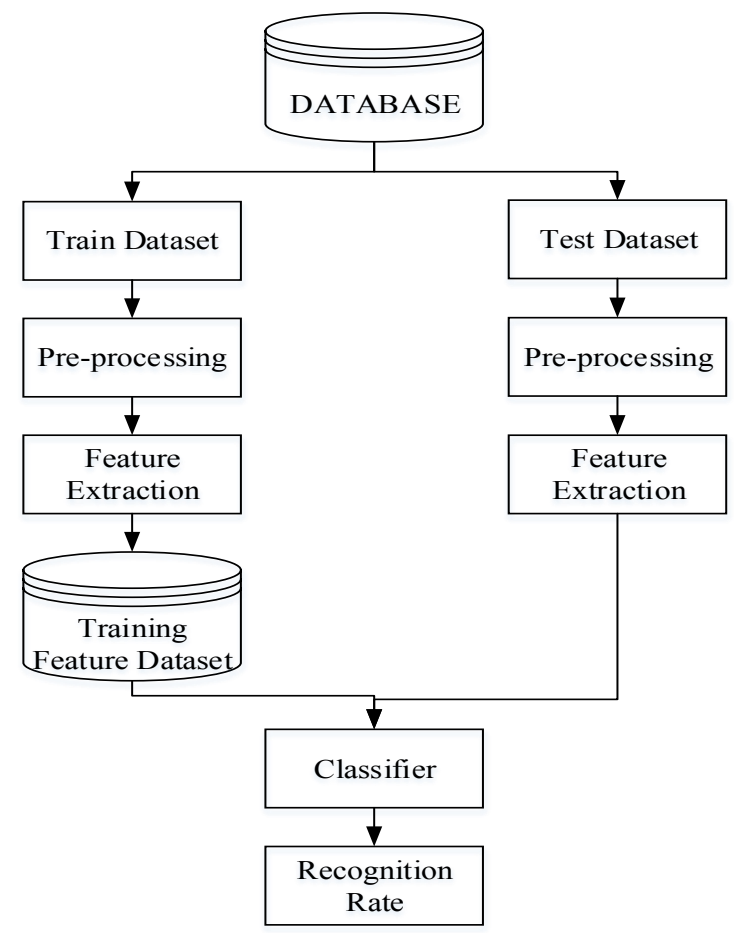

Fig. 3. Block diagram of proposed model for face recognition using SVM classifier.

\section{A. Pre-processing}

The image is collected from each face database which are of different sizes and color. In the proposed method, some of the RGB color images are converted into grayscale images then, histogram equalization is found for all the images and then resized to $90 \times 90$.

The histogram equalization is a method used for image contrast modification of an image. The contrast of an image shown in Fig. 4(a) is enhanced using histogram equalization and is shown in Fig. 4(b). The histograms of an image and enhanced contrast images are shown in Fig. 4(c) and 4(d) respectively. It is observed, that the intensity values of pixels of an image are thickly concentrated between 50 and 200, as shown in Fig. 4(c). The histogram equalization technique distributes pixel intensity values for all intensity levels i.e., between zero and 255 as shown in Fig. 4(d), hence the contrast of an image enhances.

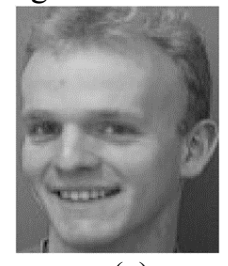

(a)

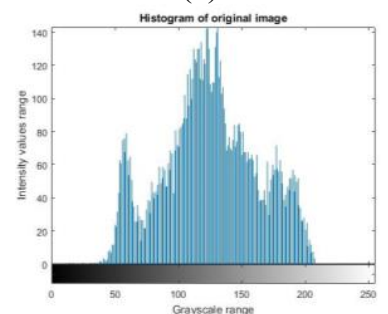

(c)

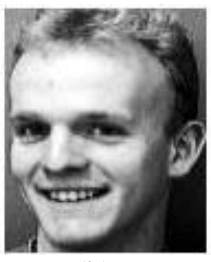

(b)

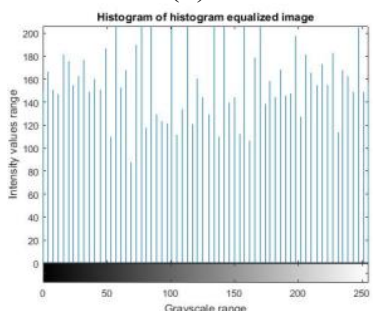

(d)
Fig. 4. (a) Original Image of face for ORL database (b) Image after histogram equalization (c) Histogram of original image and (d) Histogram of Image after histogram equalization

\section{B. Modified Local Ternary Pattern (MLTP)}

The LTP is modified by considering the threshold value as zero. The image is segmented into $3 \mathrm{X} 3$ sub blocks and then MLTP is computed using Eq. (5)

$$
T(x, y)=\left\{\begin{array}{r}
-1, P_{n}<P_{c} \\
0, P_{n}=P_{c} \\
1, P_{n}>P_{c}
\end{array}\right.
$$

where $T(x, y)=$ a modified coefficient value of neighboring pixel.

$$
\begin{aligned}
& P n=\text { the neighboring pixel value. } \\
& P c=\text { the center pixel value. }
\end{aligned}
$$

The coefficients of $T(x, y)$ are separated as (i) Left side pattern considering only negative numbers and remaining values are equated to zeros. Again negative values are converted into positive values and finally binary values are converted into decimal values. (ii) Right side pattern considering only positive numbers and remaining values are equated to zeros. Convert binary into decimal value. The 
ICIIBMS 2018, Track 1: Image Processing, Computer Science and Information technology, Bangkok, Thailand

binary numbers of left side and right side patterns are converted into decimal values starting from neighboring locations $\mathrm{T}(2,3), \mathrm{T}(1,3), \mathrm{T}(1,2), \mathrm{T}(1,1), \mathrm{T}(2,1), \mathrm{T}(3,1), \mathrm{T}$ $(3,2)$ and $T(3,3)$ in the counter-clockwise direction using Eq. (6)

$$
M L T P=\sum_{b=0}^{7} T(x, y) 2^{b}
$$

\section{Illustration of MLTP computation}

The $3 \times 3$ matrix in a face image is considered and MLTP value is computed as shown in Fig. 5a. The neighboring pixel values of 3 X3 matrix are associated with center pixel value and are assigned coefficient values based on Eq. (5). The left side and right side patterns are separated as shown in Fig. 5b. Negative values of left side pattern are converted into positive values. The binary values of left and right side pattern are converted into decimal values based on Eq. (6). The procedure is continued for all $3 \mathrm{X} 3$ matrices to convert original pixel values into MLTP coefficients. The original image size of $\mathrm{m} * \mathrm{n}$ is converted into $(\mathrm{m}-2) *(\mathrm{n}-2)$ size MLTP matrix.

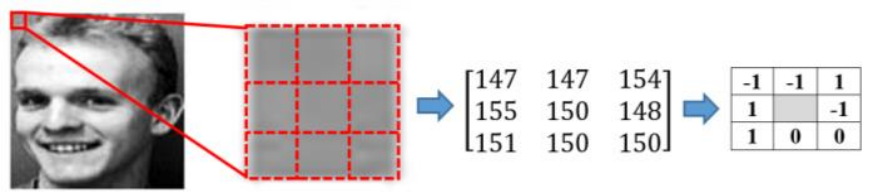

(a) 3X3 Matrix and MLTP Values

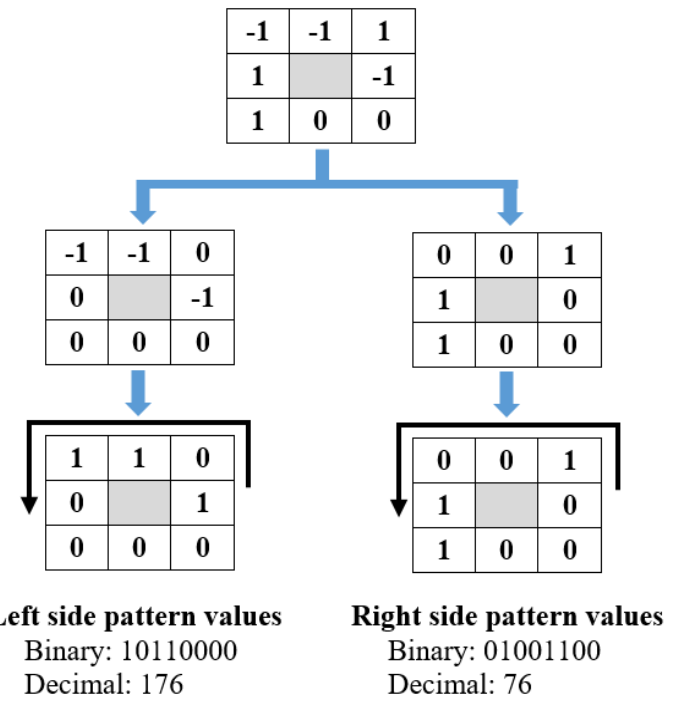

(b) Left and Right side pattern values

Fig. 5. The illustration of MLTP

MLTP is applied on face image and the corresponding left side patterns and right side patterns along with MLTP matrix values are shown in Fig. 6.
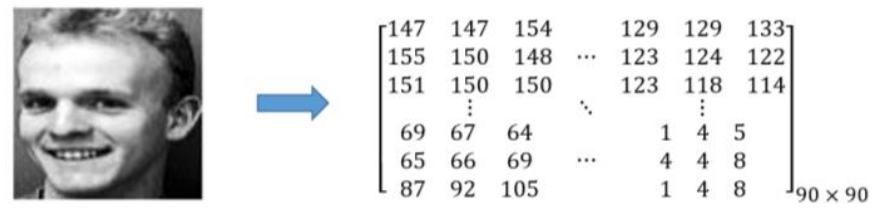

(a) The pixel value of 90X90 image size

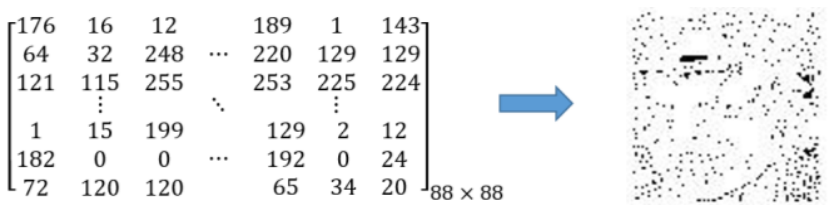

(b) The pixel values of left side pattern with an image of size 88X88

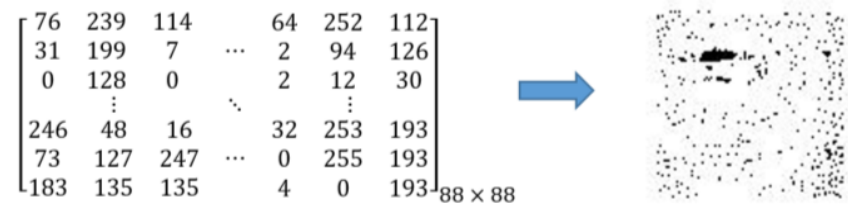

(c) The pixel values of right side pattern with an image of size 88X88

Fig. 6. The pixel values and images of left side and right side patterns

\section{Histogram on MLTP}

The left side and right side MLTP patterns are considered and histogram is applied to generate initial features. The number of MLTP coefficients of left and right side patterns are compressed during histogram and the maximum number of coefficients are 256 . In real time face recognition systems, the speed of the recognition increases rapidly due to compression with histogram.

\section{Final Features}

The histogram features of left side and right side MLTP are concatenated to extract final features. The histograms and their corresponding coefficient values are shown in Fig. 7. The numbers of final features are equated to 512 .
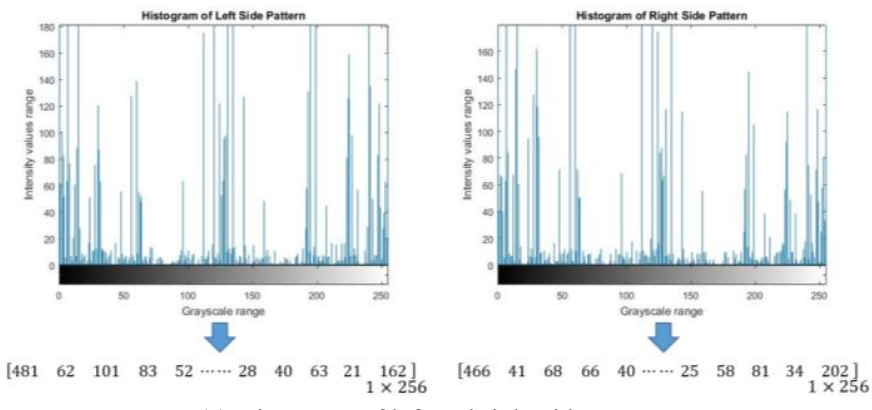

(a) Histogram of left and right side pattern

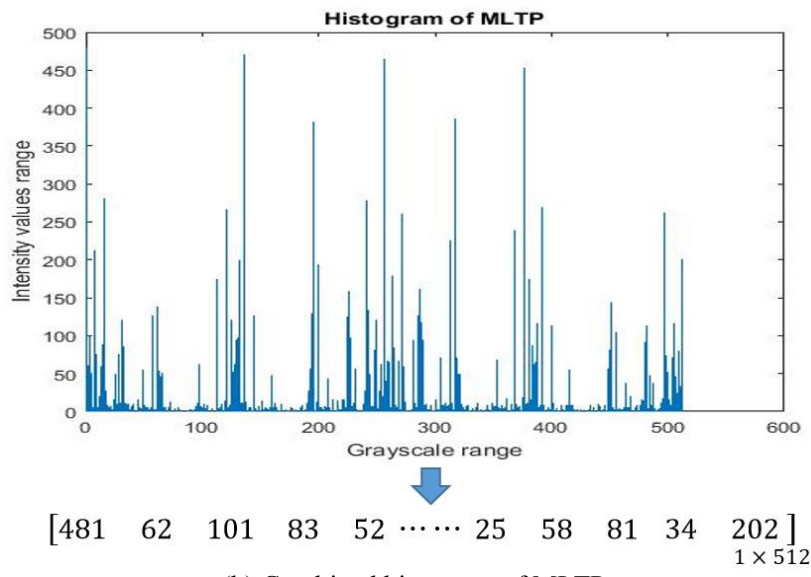

(b) Combined histogram of MLTP

Fig. 7. Histogram on MLTP

\section{- IEEE}


ICIIBMS 2018, Track 1: Image Processing, Computer Science and Information technology, Bangkok, Thailand

\section{Classification using SVM}

The face database is divided into training dataset and test dataset. The pre-processing techniques and MLTP are applied to extract the features from each image for both training and test dataset. The training feature dataset is trained by an ErrorCorrecting Output Code (ECOC) multiclass model using SVM classifier.

The training label is created in the specific location of each image of different class. The training features and training labels are trained using SVM. The obtained trained feature sets are compared with the test feature sets to predict the correct classification rate by plotting confusion matrix.

\section{PERformance ANALYsis}

In this section, definitions of performance parameters and their analysis based on recognition rate of proposed model are discussed.

\section{A. Definitions of Performance Parameter for Classifiers}

The effectiveness of the proposed model is found using the confusion matrix which is used for calculating the accuracy of the model. There are four parameters related with confusion matrix: True Positives (TP) is the case when the correct class is true and the predicted is also true, True Negatives (TN) is the case when the correct class is false and the predicted is also false, False Positive (FP) is the case when the correct class is false but the predicted is shown as true and False Negative (FN) is the case when the correct class is true but the predicted is shown as false. Then the two important parameters used to calculate the performance of the proposed model are as follows.

1) Classification Accuracy: Accuracy is the ratio of the total number of predictions that are correct and is given by Eq. 7.

$$
\text { Accuracy }=\frac{T P+T N}{T P+F P+T N+F N}
$$

2) Error Rate: Error rate is the ratio of the total number of predictions that are incorrect and is given by Eq. 8 .

$$
\text { Error Rate }=1-\text { Accuracy }=\frac{F P+F N}{T P+F P+T N+F N}
$$

\section{B. Experimental Results}

The number of training images are varied for the face databases such as ORL, YALE, Indian Females, Indian Males, JAFFE and NIR. The percentage accuracy and error rate are computed using proposed model and are tabulated in Tables I, II, III, IV, V and VI. It is shown that, as the number of training images increases, the percentage accuracy also increases. The percentage accuracy is high in the case of JAFFE face database as the variations in face images are less compared to other face databases. The percentage accuracy is less in the case of YALE face database, as variations are more in face database.

TABLE I

VARIATIONS IN ACCURACY WITH NUMBER OF TRAINING IMAGES USING ORL FACE DATABASE

\begin{tabular}{|c|c|c|c|}
\hline $\begin{array}{c}\text { Training } \\
\text { Images }\end{array}$ & Test Images & \% Accuracy & \% Error \\
\hline 240 & 160 & 83.13 & 16.87 \\
\hline 280 & 120 & 90.00 & 10.00 \\
\hline 320 & 80 & 92.50 & 7.50 \\
\hline 360 & 40 & 95.00 & 5.00 \\
\hline
\end{tabular}

TABLE II

VARIATIONS IN ACCURACY WITH NUMBER OF TRAINING IMAGES USING YALE FACE DATABASE

\begin{tabular}{|c|c|c|c|}
\hline $\begin{array}{c}\text { Training } \\
\text { Images }\end{array}$ & Test Images & \% Accuracy & \% Error \\
\hline 105 & 45 & 68.89 & 31.11 \\
\hline 120 & 30 & 73.33 & 26.67 \\
\hline 135 & 15 & 93.33 & 6.67 \\
\hline
\end{tabular}

TABLE III

VARIATIONS IN ACCURACY WITH NUMBER OF TRAINING IMAGES USING INDIAN FEMALES FACE DATABASE

\begin{tabular}{|c|c|c|c|}
\hline $\begin{array}{c}\text { Training } \\
\text { Images }\end{array}$ & Test Images & \% Accuracy & \% Error \\
\hline 154 & 88 & 95.45 & 4.55 \\
\hline 176 & 66 & 95.45 & 4.55 \\
\hline 198 & 44 & 95.45 & 4.55 \\
\hline 220 & 22 & 95.45 & 4.55 \\
\hline
\end{tabular}

TABLE IV

VARIATIONS IN ACCURACY WITH NUMBER OF TRAINING IMAGES USING INDIAN MALES FACE DATABASE

\begin{tabular}{|c|c|c|c|}
\hline $\begin{array}{c}\text { Training } \\
\text { Images }\end{array}$ & Test Images & \% Accuracy & \% Error \\
\hline 120 & 80 & 82.5 & 17.5 \\
\hline 140 & 60 & 85 & 15 \\
\hline 160 & 40 & 95 & 5 \\
\hline 180 & 20 & 96 & 4 \\
\hline
\end{tabular}

TABLE V

VARIATIONS IN ACCURACY WITH NUMBER OF TRAINING IMAGES USING JAFFE FACE DATABASE

\begin{tabular}{|c|c|c|c|}
\hline $\begin{array}{c}\text { Training } \\
\text { Images }\end{array}$ & Test Images & \% Accuracy & \% Error \\
\hline 120 & 80 & 92.5 & 7.5 \\
\hline 140 & 60 & 95.67 & 4.33 \\
\hline 160 & 40 & 96 & 4 \\
\hline 180 & 20 & 100 & 0 \\
\hline
\end{tabular}

It is observed that, as the number of training images increases, the percentage error decreases. The percentage error is more in the case of YALE face database as the variations in face images are more compared to other face databases. The percentage error is less in the case of JAFFE face database, as 
variations are less in face database. Variations in percentage accuracy and error are independent of the number of training images in case of Indian Females database.

TABLE VI

VARIATIONS IN ACCURACY WITH NUMBER OF TRAINING IMAGES USING NIR FACE DATABASE

\begin{tabular}{|c|c|c|c|}
\hline $\begin{array}{c}\text { Training } \\
\text { Images }\end{array}$ & Test Images & \% Accuracy & \% Error \\
\hline 1080 & 720 & 87.5 & 12.5 \\
\hline 1260 & 360 & 87.71 & 12.29 \\
\hline 1440 & 540 & 90.56 & 9.44 \\
\hline 1680 & 720 & 95.83 & 4.17 \\
\hline
\end{tabular}

C. Comparison of proposed algorithm with existing algorithms

The recognition rate or accuracy of the proposed method is compared with the existing methods as shown in Table VII. It is observed that the accuracy of the proposed model is $100 \%$ compared to the existing algorithms for JAFFE database and it is also observed that the proposed method achieves significantly higher accuracy compared to the popular methods used for ORL and YALE databases. The performance parameter values are computed to demonstrate that the proposed algorithm is better for the following reasons.

(i) The face images are preprocessed using histogram equalization and are resized to adjust the contrast.

(ii) The novel concept of MLTP can be used to avoid the threshold gaps in the sub blocks where all the pixels value are quite similar as in the case of LTP.

(iii) The histogram on MLTP features reduces high dimensional features to low dimensional features.

(iv) The SVM multi-class classifier is used to train the training feature vector.

TABLE VII

COMPARISON OF PROPOSED ALGORITHM WITH EXISTING ALGORITHM

\begin{tabular}{|c|l|c|}
\hline Database & \multicolumn{1}{|c|}{ Algorithm } & Accuracy (\%) \\
\hline \multirow{3}{*}{ ORL } & PCA+SVM [15] & 88.00 \\
\cline { 2 - 3 } & LTP+SVM [16] & 93.75 \\
\cline { 2 - 3 } & Proposed Algorithm & 95.00 \\
\hline \multirow{3}{*}{ YALE } & PCA+SVM [17] & 86.67 \\
\cline { 2 - 3 } & 2D-PCA + ED [18] & 92.30 \\
\cline { 2 - 3 } & Proposed Algorithm & 93.33 \\
\hline \multirow{3}{*}{ JAFFE } & PCA + ED [19] & 81.42 \\
\cline { 2 - 3 } & GWT+PCA+SVM [20] & 96.33 \\
\cline { 2 - 3 } & Proposed Algorithm & 100 \\
\hline
\end{tabular}

\section{CONCLUSION}

Biometric recognition has been getting popular in recent trends. Biometric is an automated method of recognizing a person based on his/her traits to determine the identity and authentication of a person. In this paper, we have presented a novel face recognition system with Modified Local Ternary Pattern (MLTP) using SVM classifier. In this model, the database is divided into training dataset and test dataset, and the number of training and test images are varied. In pre- processing stage, each image is converted into a gray scale, then contrast adjustment is made using histogram equalization and it is resized to $90 \times 90$ image size. Each face image is divided into 3X3 sub-block to calculate Modified Local Ternary Pattern (MLTP) for feature extraction. The final feature vector of training dataset is trained using an ECOC multiclass model with SVM classifier. The predicted output is tested with the trained feature and test feature. The experiment was conducted on six publicly used dataset and the results were tabulated. It is observed that, the proposed model has the highest accuracy for JAFEE with 180 training images, while Yale face database results in lower performance for 135 training images compared to other databases. It is also shown that the proposed method achieves enhanced results related to the popular methods. The future work includes different feature extraction techniques that can be used to reduce the calculation complexity.

\section{REFFERENCE}

[1] Naoufel Werghi, Claudio Tortorici, Stefano Berretti and Alberto Del Bimbo, "Boosting 3D LBP-Based Face Recognition by Fusing Shape and Texture Descriptors on the Mesh", IEEE Transactions on Information Forensics and Security, Vol. 11, Issue 5, pp. 964 - 979, Jan 2016

[2] Jiwen Lu, Venice Erin Liong and Jie Zhou, "Simultaneous Local Binary Feature Learning and Encoding for Homogeneous and Heterogeneous Face Recognition", IEEE Transactions on Pattern Analysis and Machine Intelligence, Vol. PP, Issue 99, pp.1-1, Aug 2017

[3] Byungyong Ryu , Adín Ramírez Rivera , Jaemyun Kim and Oksam Chae, "Local Directional Ternary Pattern for Facial Expression Recognition", IEEE Transactions on Image Processing, Vol. 26, Issue 12, pp, 6006 - 6018, July 2017

[4] Yao Wu and Weigen Qiu, "Facial Expression Recognition Based on Improved Local Ternary Pattern and Stacked Auto-Encoder", AIP Conference Proceedings, Vol. 1864, Issue 1, Aug 2017

[5] Swati Manhotra and Reecha Sharma, "Facial Feature Extraction Using Local Binary Pattern and Local Ternary Pattern with Gradient Based Illumination Normalization", Internaltional Journal of Advanced Research in Computer Science, Vol. 8, Issue 7, Aug 2017

[6] Hamdi Dibeklioglu and Theo Gevers, "Automatic Estimation of Taste Liking through Facial Expression Dynamics", IEEE Transactions on Affective Computing, pp. 1-1, May 2018

[7] Anima Majumder, Laxmidhar Behera and Venkatesh K. Subramanian, "Automatic Facial Expression Recognition System Using Deep Network-Based Data Fusion”, IEEE Transactions on Cybernetics, Vol. 48, Issue 1, pp. 103 114, Nov 2016

[8] Santosh Tirunagari , Norman Poh , David Windridge , Aamo Iorliam, Nik Suki and Anthony T. S. Ho, "Detection of Face Spoofing Using Visual Dynamics", IEEE Transactions on Information Forensics and Security, Vol. 10, Issue 4, pp. 762 - 777, Feb 2015 
ICIIBMS 2018, Track 1: Image Processing, Computer Science and Information technology, Bangkok, Thailand

[9] Shilpa Sharma and Kumud Sachdeva, "Face Recognition using PCA and SVM with Surf Technique", International Journal of Computer Applications, Vol. 129, Issue 4, pp. $41-46$, Nov 2015

[10]AT\&T Laboratories Cambridge, 'The ORL Database of Faces',1994.[Online].Available:http://www.cl.cam.ac.uk/r esearch/dtg/attarchive/facedatabase.html. [Accessed: 07July-2017].

[11] Yale University, 'The Yale Face Database', 1997. [Online].Available:http://cvc.cs.yale.edu/cvc/projects/yale faces/yalefaces.html. [Accessed: 30- July-2017].

[12] IIT Kanpur campus, 'Indian Face Databse', 2002. [Online]. Available: http://vis-www.cs.umass.edu/ vidit/ IndianFaceDatabase/. [Accessed: 15- July-2017].

[13] Michael J. Lyons, 'The Japanese Female Facial Expression (JAFFE) Database', 1998. [Online]. Available: http://www.kasrl.org/jaffe.html. [Accessed: 15- June-2017].

[14] The Biometric Research Centre (UGC/CRC), 'The Hong Kong Polytechnic University (PolyU) NIR Face Database', 2009. [Online]. Available: http://www4.comp. polyu.edu.hk/ biometrics/polyudb_face.htm. [Accessed: 07- July-2017].

[15] Min Yao and Changming Zhu, "SVM and adaboost-based classifiers with fast PCA for face reocognition", 2016 IEEE International Conference on Consumer ElectronicsChina (ICCE-China), pp.1-5, Dec 2016
[16]Prabhat Kumar Saini, Souvik Banerjee and Vaseem Durrani, " Comparative Analysis of LBP Variants in Face Recognition Application using SVM", International Journal of Innovations and Advancement in Computer Science, Vol. 6, Issue 9, pp. 316-325, Sep 2017

[17] Bhumika Pathya and Sumita Nainan, "Performance Evaluation of Face Recognition using LBP, PCA and SVM", SSRG-International Journal of Computer Science and Engineering (SSRG-IJCSE), Vol. 3, Issue 4, pp. 8588, April 2016

[18] Swarup Kumar Dandpat and Sukadev Meher, "Performance improvement for face recognition using PCA and two-dimensional PCA", In Proceedings of International Conference on Computer Communication and Informatics, Jan 2013.

[19] Mamta Santpsh and Ashok Kumar, "Superiority of PCA algorithm for Facial Expression Recognition", International Journal of Advanced Research in Computer Science, Vol.8, Issue. 3, pp. 864-868, Apr 2017.

[20] Alaa Eleyan, "Comparative Study on Facial Expression Recognition using Gabor and Dual-Tree Complex Wavelet Transforms", International Journal of Engineering and Applied Sciences (IJEAS), Vol.9, Issue. 1, pp. 1-13, 2017. 